Research Article

\title{
Some Oscillation Results of Higher-Order Linear Differential Equations with Meromorphic Coefficients
}

\author{
Zhigang Huang \\ School of Mathematics and Physics, Suzhou University of Science and Technology, Suzhou 215011, China
}

Correspondence should be addressed to Zhigang Huang, alexehuang@sina.com

Received 5 February 2012; Accepted 13 March 2012

Academic Editor: Gaston Mandata N'Guerekata

Copyright (C) 2012 Zhigang Huang. This is an open access article distributed under the Creative Commons Attribution License, which permits unrestricted use, distribution, and reproduction in any medium, provided the original work is properly cited.

We investigate the growth of solutions of higher-order nonhomogeneous linear differential equations with meromorphic coefficients. We also discuss the relationship between small functions and solutions of such equations.

\section{Introduction and Main Results}

Let $f(z)$ be a meromorphic function in the whole complex plane. Throughout this paper, we assume that the reader is familiar with the fundamental results of the Nevanlinna's value distribution theory of meromorphic functions and the standard notations such as the order $\sigma(f)$, the exponent of convergence of zero-sequence $\lambda(f)$, and the exponent of convergence of the sequence of distinct zeros $\bar{\lambda}(f)$. Moreover, a meromorphic function $\psi(z)$ is called a small function with respect to $f(z)$ if $T(r, \psi)=o(T(r, f))$ as $r \rightarrow \infty$, possible outside of a set of $r$ with finite measure, where $T(r, f)$ is the Nevanlinna characteristic function of $f(z)$. The study of oscillation of solutions of linear differential equations has attracted many interests since the work of Bank and Laine; for more details see [1]. One of the main subject of this research is the growth and zero distribution of solutions of linear differential equations. In this paper, we first discuss the growth of solutions of higher-order linear differential equation

$$
f^{(k)}+H_{k-1} f^{(k-1)}+\cdots+H_{0} f=F(z), \quad k \geq 2,
$$

where $H_{j}(j=1, \ldots, k-1), H_{0} \not \equiv 0$ and $F \not \equiv 0$ are entire functions of finite order. Some results on the growth of solutions of (1.1) have been obtained by several researchers, see [2-4]. In 
the case that the coefficients $H_{j}(j=0, \ldots, k-1)$ are polynomials, the growth of solutions of (1.1) has been extensively studied; see [2]. In 1992, Hellerstein et al. [3] proved that every transcendental solution of (1.1) is of infinite order, if there exists some $d \in\{0,1, \ldots, k-1\}$ such that $\max _{j \neq d}\left\{\sigma\left(H_{j}\right), \sigma(F)\right\}<\sigma\left(H_{d}\right) \leq 1 / 2$. As for sectorial growth conditions on the coefficients of (1.1) that imply that all solutions are of infinite order, see, for example, [5]. In addition, a special case for this was investigated by Wang and Laine [6]. Recently, Wang and Laine [4] obtain the following.

Theorem A. Suppose that $H_{j}(z)=D_{j}(z) e^{P_{j}(z)}(j=0, \ldots, k-1)$ where $P_{j}(z)=a_{j n} z^{n}+\cdots+a_{j 0}(j=$ $0, \ldots, k-1)$ are polynomials with degree $n \geq 1, D_{j}(z)$ are entire functions of order less than $n$, not all vanishing, and $F(z) \neq \equiv$ is an entire function of order less than $n$. If $a_{j n}(j=0, \ldots, k-1)$ are distinct complex numbers, then every solution of (1.1) is of infinite order.

Thus a natural question is whether every meromorphic solution of (1.1) has infinite order, if the coefficients of (1.1) are meromorphic? The following theorem partially answers this question.

Theorem 1.1. Let $A_{j}(z)(j=1, \ldots, k-1), A_{0} \not \equiv 0$ and $F \not \equiv 0$ be meromorphic functions with $\left.\max \left\{\sigma\left(A_{j}\right), j=0,1, \ldots, k-1\right), \sigma(F)\right\}<1$, and let $a_{0}, a_{1}, \ldots, a_{k-1}$ be complex constants such that (i) $\arg a_{j}=\arg a_{0}$ and $a_{j}=c_{j} a_{0}\left(0<c_{j}<1\right)$ or (ii) $\arg a_{j} \neq \arg a_{0}(j=1, \ldots, k-1)$. Then every meromorphic solution $f \not \equiv 0$ of the equation

$$
f^{(k)}+A_{k-1} e^{a_{k-1} z} f^{(k-1)}+\cdots+A_{0} e^{a_{0} z} f=F, \quad k \geq 2,
$$

satisfies $\lambda(f)=\bar{\lambda}(f)=\sigma(f)=\infty$.

Remark 1.2. In Theorem 1.1, if $a_{j} z(j=0, \ldots, k-1)$ are replaced by $P_{j}(z)=a_{j n} z^{n}+\cdots+a_{j 0}(j=$ $0, \ldots, k-1)$, with additional hypothesis on $a_{j n}$ similar to $a_{j}$, and furthermore $\sigma(F)$ is replaced by $\sigma(F)<n$, then we have the same conclusion with Theorem 1.1.

In 2000, Chen [7] first studied the fixed points of solutions of second-order linear differential equations and obtained some precise estimation on the number of fixed points. After that, a number of results on fixed points of solutions of differential equations with entire coefficients were obtained; see [8-10]. In 2006, Chen and Shon [11] further studied the relation between small functions and solutions of differential equations and obtain the following.

Theorem B. Let $A_{j}(z) \not \equiv 0(j=0,1)$ be entire functions with $\sigma\left(A_{j}\right)<1$, and let $a, b$ be complex constants such that $a b \neq 0$ and $\arg a \neq \arg b$ or $a=c b(0<c<1)$. If $\psi(z) \neq \equiv$ is an entire function with finite order, then every solution $f \not \equiv 0$ of equation

$$
f^{\prime \prime}+A_{1}(z) e^{a z} f^{\prime}+A_{0}(z) e^{b z} f=0
$$

satisfies $\bar{\lambda}(f-\psi)=\bar{\lambda}\left(f^{\prime}-\psi\right)=\bar{\lambda}\left(f^{\prime \prime}-\psi\right)=\infty$.

Motivated by Theorem B, we try to consider the relation between small functions with meromorphic solutions of (1.2). Indeed, such relationship on higher order differential equations is more difficult than that of second order differential equations. Moreover, the 
method used in the proof of Theorem B cannot deal with the case of higher-order linear differential equations.

Theorem 1.3. Under the assumption of Theorem 1.1, if $\phi \not \equiv 0$ is a meromorphic function of finite order and satisfies $\max \{\lambda(\phi), \lambda(1 / \phi)\}<1$, then every non-trivial meromorphic solution of (1.2) satisfies $\bar{\lambda}(f-\phi)=\bar{\lambda}\left(f^{\prime}-\phi\right)=\infty$.

Remark 1.4. As the remark on Theorem 1.1 in Remark 1.2, the conclusion of Theorem 1.3 can also be extended to the case that $a_{j} z(j=0, \ldots, k-1)$ are replaced by $P_{j}(z)=a_{j n} z^{n}+\cdots+$ $a_{j 0}(j=0, \ldots, k-1)$, with some similar additional hypothesis on $a_{j n}, F$ and $\phi$ in Theorem 1.3.

\section{Preliminary Lemmas}

To prove our theorems, we need some lemmas.

Lemma 2.1 (see [12]). Let $w(z)$ be a transcendental meromorphic function with $\sigma(f)=\sigma<\infty$. Let $\Gamma=\left\{\left(k_{1}, j_{1}\right), \ldots,\left(k_{m}, j_{m}\right)\right\}$ be a finite set of distinct pairs of integers satisfying $k_{i}>j_{i} \geq 0$ for $i=1,2, \ldots, m$. Also let $\epsilon>0$ be a given constant. Then, there exists a set $E_{1} \subset(1, \infty)$ that has finite logarithmic measure, such that for all $z$ satisfying $z \notin E \cup[0,1]$ and for all $(k, j) \in \Gamma$, One has

$$
\frac{\left|w^{(k)}(z)\right|}{\left|w^{(j)}(z)\right|} \leq|z|^{(k-j)(\sigma-1+\varepsilon)} .
$$

Let $P(z)=(\alpha+\beta i) z^{n}+\cdots$ is a nonconstant polynomial and $\alpha, \beta$ are real constants. For $\theta \in[0,2 \pi)$, set $\delta(P(z), \theta)=\alpha \cos n \theta-\beta \sin n \theta$.

Lemma 2.2 (see [13]). Let $P(z)$ be a non-constant polynomial of degree $n$. Let $w(z)$ be a meromorphic function, not identically zero, of order less than $n$, and set $g(z)=w(z) e^{P(z)}$. Then for any given $\varepsilon>0$ there exists a zero measure set $H_{1} \subset[0,2 \pi)$ such that if $\theta \in \theta \in[0,2 \pi) \backslash\left(H_{1} \cup H_{2}\right)$, then for $|z|>r(\theta)$.

(1) If $\delta(P, \theta)<0$, then $\exp \left((1+\epsilon) \delta(P, \theta) r^{n}\right) \leq\left|g\left(r e^{i \theta}\right)\right| \leq \exp \left((1-\epsilon) \delta(P, \theta) r^{n}\right)$;

(2) If $\delta(P, \theta)>0$, then $\exp \left((1-\epsilon) \delta(P, \theta) r^{n}\right) \leq\left|g\left(r e^{i \theta}\right)\right| \leq \exp \left((1+\epsilon) \delta(P, \theta) r^{n}\right)$, where $H_{2}=\{\theta: \delta(P, \theta)=0,0 \leq \theta<2 \pi\}$ is a finite set.

Lemma 2.3 (see [14]). Let $A_{0}, \ldots, A_{k-1}, F \neq \equiv 0$ be finite-order meromorphic functions. If $f$ is an infinite-order meromorphic solution of the equation

$$
f^{(k)}+A_{k-1} f^{(k-1)}+\cdots+A_{0} f=F
$$

then $f$ satisfies $\lambda(f)=\bar{\lambda}(f)=\sigma(f)=\infty$.

Lemma 2.4 (see [15], page 79). Suppose that $f_{1}(z), f_{2}(z), \ldots, f_{n}(z)(n \geq 2)$ are meromorphic functions and $g_{1}(z), g_{2}(z), \ldots, g_{n}(z)$ are entire functions satisfying the following conditions:

(1) $\sum_{j=1}^{n} f_{j}(z) e^{g_{j}(z)} \equiv 0$;

(2) $g_{j}(z)-g_{k}(z)$ are not constants for $1 \leq j<k \leq n$; 
(3) For $1 \leq j \leq n, 1 \leq h<k \leq n, T\left(r, f_{j}\right)=o\left\{T\left(r, e^{g_{h}-g_{k}}\right)\right\}(r \rightarrow \infty, r \notin E)$, where E has a finite measure.

Then $f_{j}(z) \equiv 0(j=1,2, \ldots, n)$.

Lemma 2.5 (see [4], Lemma 2.6). Let $f(z)$ be a an entire function of order $\sigma(f)=\sigma<\infty$. Suppose that there exists a set $E \subset[0,2 \pi)$ which has linear measure zero, such that $\log ^{+}\left|f\left(r e^{i \theta}\right)\right| \leq M r^{\rho}$ for any ray $\arg z=\theta \in[0,2 \pi) \backslash E$, where $M$ is a positive constant depending on $\theta$, while $\rho$ is a positive constant independent of $\theta$. Then $\sigma(f) \leq \rho$.

Lemma 2.6 (see [16]). Let $f(z)$ be a transcendental meromorphic function of order $\sigma(f)=\sigma<\infty$. Then for any given $\varepsilon>0$, there exists a set $E_{3} \subset[0,2 \pi)$ which has linear measure zero, such that if $\psi_{1} \in[0,2 \pi) \backslash E_{3}$, then there is a constant $R_{1}=R_{1}\left(\psi_{1}\right)>1$ such that for all $z$ satisfying $\arg z=\psi_{1}$ and $|z|=r \geq R_{2}$, One has

$$
\exp \left\{-r^{\sigma+\varepsilon}\right\} \leq|f(z)| \leq \exp \left\{r^{\sigma+\varepsilon}\right\}
$$

\section{Proof of Theorem 1.1}

Assume that $f(z)$ is a meromorphic solution of (1.2) of finite order. We will deduce a contradiction later. First, we have $\sigma(f) \geq 1$. Otherwise, it follows from Lemma 2.4 that $A_{0} \equiv 0$ which is a contradiction.

Since $f$ is a meromorphic solution of (1.2), we know that the poles of $f$ can occur only at the poles of $A_{j}(j=0, \ldots, k-1)$ and $F$. Let $f=g(z) / d(z)$, where $d(z)$ is the canonical product formed with the nonzero poles of $f(z)$, with $\sigma(d) \leq \max \left\{\sigma(F), \sigma\left(A_{j}\right), j=0, \ldots, k-\right.$ $1\}<1$, and $g$ is an entire function with $1 \leq \sigma(g)=\sigma(f)=\sigma<\infty$. Substituting $f=g / d$ into (1.2), by some calculation we can get

$$
\begin{aligned}
d F=g^{(k)} & +g^{(k-1)}\left[A_{k-1} e^{a_{k-1} z}+B_{k, k-1}\right]+\cdots \\
& +g^{\prime}\left[A_{1} e^{a_{1} z}+\sum_{i=2}^{k-1} A_{i} e^{a_{i} z} B_{i, 1}+B_{k, 1}\right]+g\left[A_{0} e^{a_{0} z}+\sum_{i=1}^{k-1} A_{i} e^{a_{i} z} B_{i, 1}+B_{k, 0}\right]
\end{aligned}
$$

where $B_{i, j}$ are defined as a sum of a finite number of terms of the type

$$
\sum_{\left(j_{1} \cdots j_{i}\right)} C_{j j_{1} \cdots j_{i}}\left(\frac{d^{\prime}}{d}\right)^{j_{1}} \cdots\left(\frac{d^{(i)}}{d}\right)^{j_{i}}
$$

$C_{j j_{1} \cdots j_{i}}$ are constants, and $j+j_{1}+2 j_{2}+\cdots+i j_{i}=k$. Now, we rewrite (3.1) into

$$
\frac{d F}{g}=\frac{g^{(k)}}{g}+\cdots+\left[A_{1} e^{a_{1} z}+\sum_{i=2}^{k-1} A_{i} e^{a_{i} z} B_{i, 1}+B_{k, 1}\right] \frac{g^{\prime}}{g}+\left[A_{0} e^{a_{0} z}+\sum_{i=1}^{k-1} A_{i} e^{a_{i} z} B_{i, 1}+B_{k, 0}\right]
$$


Set $\max \left\{\sigma\left(A_{j}\right), \sigma(F), \sigma(d), j=0, \ldots, k-1\right\}=\beta$. By Lemma 2.1, for any given $\varepsilon>0$ there exists a set $E_{1} \in[0,2 \pi)$ which has linear measure zero, such that if $\theta \in[0,2 \pi) \backslash E_{1}$, then there is a constant $R_{1}=r_{1}(\theta)>1$ such that for all $z$ satisfying $\arg z=\theta$ and $|z| \geq R_{1}$, we have

$$
\frac{\left|g^{(i)}(z)\right|}{|g(z)|} \leq|z|^{k(\sigma-1+\varepsilon)}, \quad \frac{\left|d^{(i)}(z)\right|}{|d(z)|} \leq|z|^{k(\beta-1+\varepsilon)}, \quad i=1, \ldots, k .
$$

Meanwhile, by Lemma 2.6, for the above $\varepsilon$ there exists a set $E_{2}$ of zero linear measure, such that if $\theta \in[0,2 \pi) \backslash E_{2}$, we have for sufficiently large $r=|z|$,

$$
\left|A_{j}(z)\right| \leq \exp \left\{r^{\beta+\varepsilon}\right\}, \quad j=0, \ldots, k-1 .
$$

Next we divide our proof into two cases.

Case 1. Suppose that $\arg a_{j}=\arg a_{0}$ and $a_{j}=c_{j} a_{0}\left(0<c_{j}<1\right), j=1, \ldots, k-1$. Then for any ray $\arg z=\theta$, we have

$$
\delta\left(a_{j} z, \theta\right)=c_{j} \delta\left(a_{0} z, \theta\right)
$$

By Lemma 2.2, for any given $\varepsilon$ we can find a ray arg $z=\theta \in[0,2 \pi) \backslash E_{1} \cup E_{2} \cup H_{1} \cup H_{2}$, where $H_{1}$ and $H_{2}$ are defined in Lemma 2.2, $E_{1} \cup E_{2} \cup H_{1} \cup H_{2}$ has zero linear measure, such that $\delta\left(a_{j} z, \theta\right)=c_{j} \delta\left(a_{0} z, \theta\right)>0$, and for sufficiently large $r$,

$$
\begin{aligned}
&\left|A_{0}\left(r e^{i \theta}\right) e^{a_{0} r e^{i \theta}}\right| \geq \exp \left\{(1-\varepsilon) \delta\left(a_{0} z, \theta\right) r\right\}, \\
&\left|A_{j}\left(r e^{i \theta}\right) e^{a_{j} r e^{i \theta}}\right| \leq \exp \left\{(1+\varepsilon) c_{j} \delta\left(a_{0} z, \theta\right) r\right\}, \quad j=1, \ldots, k-1 .
\end{aligned}
$$

Thus, by (3.4) and (3.7), we have for sufficiently large $r$,

$$
\begin{gathered}
\left|A_{0} e^{a_{0} z}+\sum_{i=1}^{k-1} A_{i} e^{a_{i} z} B_{i, 1}+B_{k, 0}\right| \geq M_{0} \exp \left\{(1-\varepsilon) \delta\left(a_{0} z, \theta\right) r\right\} \\
\left|A_{k-1} e^{a_{k-1} z}+B_{k, k-1}\right| \leq M_{k-1} \exp \left\{(1-\varepsilon) \delta\left(a_{j} z, \theta\right) r\right\}=M_{k-1} \exp \left\{(1-\varepsilon) c_{k-1} \delta\left(a_{0} z, \theta\right) r\right\}, \\
\cdots \\
\left|A_{1} e^{a_{1} z}+\sum_{i=2}^{k-1} A_{i} e^{a_{i} z} B_{i, 1}+B_{k, 1}\right| \leq M_{1} \exp \left\{(1+\varepsilon) \delta\left(a_{1} z, \theta\right) r\right\}=M_{1} \exp \left\{(1+\varepsilon) c_{1} \delta\left(a_{0} z, \theta\right) r\right\},
\end{gathered}
$$

where $M_{j}(j=0, \ldots, k-1)$ are positive constants.

For $\theta \in[0,2 \pi) \backslash E_{1} \cup E_{2} \cup H_{1} \cup H_{2}$, we claim that

$$
\frac{\log ^{+}|g(z)|}{|z|^{\beta+\varepsilon}}
$$


is bounded on the ray $\arg z=\theta$. Otherwise, there exists a sequence of points $z_{m}=r_{m} e^{i \theta}$, such that $r_{m} \rightarrow \infty$, and that

$$
\frac{\log ^{+}\left|g\left(z_{m}\right)\right|}{r_{m}^{\beta+\varepsilon}} \longrightarrow \infty
$$

From (3.10) and the definition of order, we see that

$$
\left|\frac{d\left(z_{m}\right) F\left(z_{m}\right)}{g\left(z_{m}\right)}\right| \longrightarrow 0
$$

for $m$ is large enough.

By (3.3),(3.4), and (3.8)-(3.11), we get for sufficiently large $m$,

$$
\begin{aligned}
M_{0} \exp \left\{(1-\varepsilon) \delta\left(a_{0} z, \theta\right) r_{m}\right\} \leq & \left|A_{0} e^{a_{0} r_{m} e^{i \theta}}+\sum_{i=1}^{k-1} A_{i} e^{a_{i} r_{m} e^{i \theta}} B_{i, 1}+B_{k, 0}\right| \\
\leq & \left|-\frac{d\left(r_{m} e^{i \theta}\right) F\left(r_{m} e^{i \theta}\right)}{g}\right|+\left|\frac{g^{(k)}}{g}\right| \\
& +\cdots+\left|\left[A_{1} e^{a_{1} r_{m} e^{i \theta}}+\sum_{i=2}^{k-1} A_{i} e^{a_{i} r_{m} e^{i \theta}} B_{i, 1}+B_{k, 1}\right] \frac{g^{\prime}}{g}\right| \\
\leq & \sum_{j=1}^{k-1} r^{k(\sigma+\varepsilon)} M_{j} \exp \left\{(1+\varepsilon) c_{j} \delta\left(a_{0} z, \theta\right) r_{m} e^{i \theta}\right\}+r^{k(\sigma-1+\varepsilon)} .
\end{aligned}
$$

Clearly, we can choose sufficiently small $\varepsilon$ such that $0<\varepsilon<\min \left\{\left(1-c_{j}\right) /\left(1+c_{j}\right): j=\right.$ $1, \ldots, k-1\}$. Then by (3.12), we can obtain a contradiction provided that $m$ is sufficiently large.

Therefore,

$$
\frac{\log ^{+}|g(z)|}{|z|^{\beta+\varepsilon}}
$$

is bounded, and we have $|g(z)| \leq M \exp \left\{r^{\beta+\varepsilon}\right\}$ on the ray $\arg z=\theta$.

Case 2. Suppose that $\arg a_{j} \neq \arg a_{0}(j=1, \ldots, k-1)$. By Lemma 2.2, there exists a ray $\arg z=$ $\theta \in[0,2 \pi) \backslash E_{1} \cup E_{2} \cup H_{1} \cup H_{2}$, where $E_{1}, E_{2}, H_{1}$, and $H_{2}$ are defined, respectively, as in Case 1 , such that

$$
\delta\left(a_{0} z, \theta\right)>0, \quad \delta\left(a_{j} z, \theta\right)<0, \quad j=1, \ldots, k-1 .
$$


Further, for any given $\varepsilon>0$, we have for sufficiently large $r$,

$$
\begin{aligned}
&\left|A_{0}\left(r e^{i \theta}\right) e^{a_{0} r e^{i \theta}}\right| \geq \exp \left\{(1-\varepsilon) \delta\left(a_{0} z, \theta\right) r\right\}, \\
&\left|A_{j}\left(r e^{i \theta}\right) e^{a_{j} r e^{i \theta}}\right| \leq \exp \left\{(1-\varepsilon) \delta\left(a_{j} z, \theta\right) r\right\}, \quad j=1, \ldots, k-1 .
\end{aligned}
$$

Thus, by (3.4) and (3.15), we have for sufficiently large $r$,

$$
\begin{aligned}
\left|A_{0} e^{a_{0} z}+\sum_{i=1}^{k-1} A_{i} e^{a_{i} z} B_{i, 1}+B_{k, 0}\right| & \geq M_{0} \exp \left\{(1-\varepsilon) \delta\left(a_{0} z, \theta\right) r\right\}, \\
\left|A_{k-1} e^{a_{k-1} z}+B_{k, k-1}\right| & \leq M_{k-1} r^{k(\sigma-1+\varepsilon)}, \\
\cdots & \\
\left|A_{1} e^{a_{1} z}+\sum_{i=2}^{k-1} A_{i} e^{a_{i} z} B_{i, 1}+B_{k, 1}\right| & \leq M_{1} r^{k(\sigma-1+\varepsilon)},
\end{aligned}
$$

where $M_{j}(j=0, \ldots, k-1)$ are positive constants.

For $\theta \in[0,2 \pi) \backslash E_{1} \cup E_{2} \cup H_{1} \cup H_{2}$, we claim that

$$
\frac{\log ^{+}|g(z)|}{|z|^{\beta+\varepsilon}}
$$

is bounded on the ray $\arg z=\theta$. Otherwise, there exists a sequence of points $z_{m}=r_{m} e^{i \theta}$, such that $r_{m} \rightarrow \infty$, and that

$$
\frac{\log ^{+}\left|g\left(z_{m}\right)\right|}{r_{m}^{\beta+\varepsilon}} \longrightarrow \infty
$$

From (3.18) and the definition of order, we see that

$$
\left|\frac{d\left(z_{m}\right) F\left(z_{m}\right)}{g\left(z_{m}\right)}\right| \rightarrow 0
$$

for $m$ is large enough.

Then by similar reasoning as in Case 1, we can also obtain a contradiction. So

$$
\frac{\log ^{+}|g(z)|}{|z|^{\beta+\varepsilon}}
$$

is bounded, and we have $|g(z)| \leq M \exp \left\{r^{\beta+\varepsilon}\right\}$ on the ray $\arg z=\theta$.

Combining Case 1 and Case 2, for any given ray $\arg z=\theta \in[0,2 \pi) \backslash E, E$ of linear measure zero, we have $|g(z)| \leq M \exp \left\{r^{\beta+\varepsilon}\right\}$ on the ray $\arg z=\theta$, provided that $r$ is sufficiently large. Thus by Lemma 2.5 , we get $\sigma(g) \leq \beta+\varepsilon<1$, which is a contradiction. Again by Lemma 2.3, we complete the proof of Theorem 1.1. 


\section{Proof of Theorem 1.3}

Let $f(z) \not \equiv 0$ be a meromorphic solution of (1.2). Set $B_{j}(z)=A_{j}(z) e^{a_{j} z}$. First, we prove that $\bar{\lambda}(f-\phi)=\infty$.

Set $f=g_{0}+\phi$. By Theorem 1.1, we have $\sigma\left(g_{0}\right)=\sigma(f)=\infty$. Substituting $f=g_{0}+\phi$ into (1.2), we get

$$
g_{0}^{(k)}+B_{k-1} g_{0}^{(k-1)}+\cdots+B_{0} g_{0}=F-\left(\phi^{(k)}+B_{k-1} \phi^{(k-1)}+\cdots+B_{0} \phi\right) .
$$

We remark that (4.1) may have finite-order solutions, but we only need to discuss the solutions whose order are $\infty$.

If $\phi^{(k)}+B_{k-1} \phi^{(k-1)}+\cdots+B_{0} \phi=F$, then by Theorem 1.1, we have $\sigma(\phi)=\infty$. It is a contradiction. Hence we have $F-\left(\phi^{(k)}+B_{k-1} \phi^{(k-1)}+\cdots+B_{0} \phi\right) \not \equiv 0$. It follows from Lemmas 3 and (4.1) that $\bar{\lambda}\left(g_{0}\right)=\lambda\left(g_{0}\right)=\sigma\left(g_{0}\right)=\infty$.

Now we prove that $\bar{\lambda}\left(f^{\prime}-\phi\right)=\infty$. Set $g_{1}=f^{\prime}-\phi$. Differentiating both side of (1.2), we get

$$
f^{(k+1)}+B_{k-1} f^{(k)}+\left(B_{k-1}^{\prime}+B_{k-2}\right) f^{(k-1)}+\cdots+\left(B_{1}^{\prime}+B_{0}\right) f^{\prime}+B_{0}^{\prime} f=F^{\prime}
$$

Rewriting (1.2), we have

$$
f=F-\frac{1}{B_{0}}\left[f^{(k)}+B_{k-1} f^{(k-1)}+\cdots+B_{1} f^{\prime}\right]
$$

Substituting (4.3) into (4.2), we get

$$
\begin{aligned}
f^{(k+1)}+ & {\left[B_{k-1}-\frac{B_{0}^{\prime}}{B_{0}}\right] f^{(k)}+\left[B_{k-1}^{\prime}+B_{k-2}-\frac{B_{0}^{\prime}}{B_{0}} B_{k-1}\right] f^{(k-1)} } \\
& +\cdots+\left[B_{1}^{\prime}+B_{0}-\frac{B_{0}^{\prime}}{B_{0}} B_{1}\right] f^{\prime}=F^{\prime}-B_{0}^{\prime} F .
\end{aligned}
$$

We rewrite (4.4) into the following form:

$$
f^{(k+1)}+C_{k-1} f^{(k)}+C_{k-2} f^{(k-1)}+\cdots+C_{0} f^{\prime}=F^{\prime}-B_{0}^{\prime} F,
$$

where $C_{k-1}=B_{k-1}-B_{0}^{\prime} / B_{0}, C_{j}=B_{j+1}^{\prime}+B_{j}-\left(B_{0}^{\prime} / B_{0}\right) B_{j+1}, j=0, \ldots, k-2$. Substituting $f^{\prime}=$ $g_{1}+\phi, \ldots, f^{(k+1)}=g_{1}^{(k)}+\phi^{(k)}$ into (4.5), we have

$$
\begin{aligned}
g_{1}^{(k)} & +C_{k-1} g_{1}^{(k-1)}+C_{k-2} g_{1}^{(k-2)}+\cdots+C_{0} g_{1} \\
& =F^{\prime}-B_{0}^{\prime} F-\left[\phi^{(k)}+C_{k-1} \phi^{(k-1)}+C_{k-2} \phi^{(k-2)}+\cdots+C_{0} \phi\right] .
\end{aligned}
$$


Abstract and Applied Analysis

Next we prove

$$
Q_{1}(z)=\phi^{(k)}+C_{k-1} \phi^{(k-1)}+C_{k-2} \phi^{(k-2)}+\cdots+C_{0} \phi-F^{\prime}+B_{0}^{\prime} F \not \equiv 0
$$

Conversely, assume that $Q_{1}(z) \equiv 0$.

Dividing $\phi$ into both sides of $Q_{1}(z) \equiv 0$, we have

$$
D_{k-1} e^{a_{k-1} z}+\cdots+D_{1} e^{a_{1} z}+D_{0} e^{a_{0} z}+D(z) \equiv 0,
$$

where

$$
\begin{aligned}
& D_{0}=A_{0}+A_{0}^{\prime} F+a_{0} A_{0} F, \\
& D_{1}=A_{1} \frac{\phi^{\prime}}{\phi}+\left(A_{1}^{\prime}+A_{1} a_{1}-\frac{A_{0}^{\prime}}{A_{0}} A_{1}\right), \\
& D_{2}=A_{2} \frac{\phi^{\prime \prime}}{\phi}+\left(A_{2}^{\prime}+A_{2} a_{2}-\frac{A_{0}^{\prime}}{A_{0}} A_{2}\right) \frac{\phi^{\prime}}{\phi}, \\
& \ldots \\
& D_{j}=A_{j} \frac{\phi^{(j)}}{\phi}+\left(A_{j}^{\prime}+A_{j} a_{j}-\frac{A_{0}^{\prime}}{A_{0}} A_{j}\right) \frac{\phi^{(j-1)}}{\phi}, \\
& \quad \ldots \\
& D_{k-1}=A_{k-1} \frac{\phi^{(k-1)}}{\phi}+\left(A_{k-1}^{\prime}+A_{k-1} a_{k-1}-\frac{A_{0}^{\prime}}{A_{0}} A_{k-1}\right) \frac{\phi^{(k-2)}}{\phi}, \\
& D=\frac{\phi^{(k)}}{\phi}-\frac{A_{0}^{\prime}}{A_{0}} \frac{\phi^{(k-1)}}{\phi}-F^{\prime} .
\end{aligned}
$$

Set $\max \left\{\sigma\left(A_{j}\right), \sigma(F), j=0, \ldots, k-1\right\}=\beta$. Clearly from (4.9),

$$
\sigma\left(D_{0}(z)\right) \leq \beta
$$

Meanwhile, by (4.9), we have

$$
\begin{gathered}
m\left(r, D_{j}\right) \leq 3 m\left(r, A_{j}\right)+m\left(r, \frac{\phi^{(j)}}{\phi}\right)+m\left(r, \frac{\phi^{(j-1)}}{\phi}\right)+m\left(r, A_{j}^{\prime}\right)+m\left(r, \frac{A_{0}^{\prime}}{A_{0}}\right)+O(1) \\
\leq 4 m\left(r, A_{j}\right)+O(\log r), \quad j=1, \ldots, k-1, \\
m(r, D) \leq m\left(r, F^{\prime}\right)+O(\log r)
\end{gathered}
$$


outside a possible exceptional set $E$ of finite linear measure. On the other hand, again by (4.9), we have

$$
\begin{array}{r}
N\left(r, D_{j}\right) \leq N\left(r, \frac{1}{\phi}\right)+N\left(r, \frac{1}{A_{0}}\right)+k\left(N\left(r, A_{0}\right)+N(r, \phi)+N\left(r, A_{j}\right)\right), \\
j=1, \ldots, k-1, \\
N(r, D) \leq N\left(r, \frac{1}{\phi}\right)+N\left(r, \frac{1}{A_{0}}\right)+2 N\left(r, A_{0}\right)+k N(r, \phi)+N\left(r, F^{\prime}\right) .
\end{array}
$$

Combining (4.11) and (4.12), we get

$$
\begin{gathered}
\sigma\left(D_{j}\right) \leq \max \left\{\lambda(\phi), \lambda\left(\frac{1}{\phi}\right), \sigma\left(A_{j}\right), j=0, \ldots, k-1\right\}<1, \quad j=1, \ldots, k-1, \\
\sigma(D) \leq \max \left\{\lambda(\phi), \lambda\left(\frac{1}{\phi}\right), \sigma\left(F^{\prime}\right), \sigma\left(A_{0}\right)\right\}<1 .
\end{gathered}
$$

By Lemma 2.6, for any given positive constants $\varepsilon$, there exists a ray $\arg z=\theta \in[0,2 \pi) \backslash E_{1}$, where $E_{1}$ has zero linear measure, such that for sufficiently large $r$,

$$
|D(z)| \leq \exp \left\{r^{\sigma(D)+\varepsilon}\right\}
$$

Therefore by (4.13), and Lemma 2.2, we can find a ray $\arg z=\theta \in[0,2 \pi) \backslash E_{1} \cup H_{1} \cup H_{2}$, where $H_{1}$ and $H_{2}$ are defined in Lemma 2.2, $H_{1} \cup H_{2}$ has zero linear measure, such that $\delta\left(a_{j} z, \theta\right)=c_{j} \delta\left(a_{0} z, \theta\right)>0$, and for sufficiently large $r$,

$$
\begin{gathered}
\left|D_{0}\left(r e^{i \theta}\right) e^{a_{0} r e^{i \theta}}\right| \geq \exp \left\{(1-\varepsilon) \delta\left(a_{0} z, \theta\right) r\right\}, \\
\left|D_{j}\left(r e^{i \theta}\right) e^{a_{j} r e^{i \theta}}\right| \leq \exp \left\{(1+\varepsilon) c_{j} \delta\left(a_{0} z, \theta\right) r\right\}, \quad j=1, \ldots, k-1 .
\end{gathered}
$$

Thus, by (4.8) and (4.14) and (4.15), we have

$$
\exp \left\{(1-\varepsilon) \delta\left(a_{0} z, \theta\right) r\right\} \leq \sum_{j=1}^{k-1} \exp \left\{(1+\varepsilon) c_{j} \delta\left(a_{0} z, \theta\right) r\right\}+\exp \left\{r^{\sigma(D)+\varepsilon}\right\}
$$

Since $\varepsilon$ can be chosen arbitrarily small, we can obtain a contradiction. Thus we have $Q_{1}(z) \not \equiv 0$. By Lemma 2.3 and (4.6), we get $\bar{\lambda}\left(f^{\prime}-\phi\right)=\infty$.

\section{Acknowledgments}

The author would like to thank the referee for his/her valuable suggestions and comments. This research is supported by NNSF of China (no. 11001057), NSF of Jiangsu Province (BK2010234), Project of Qinglan of Jiangsu Province. 


\section{References}

[1] I. Laine, Nevanlinna Theory and Complex Differential Equations, vol. 15, Walter de Gruyter \& Co., Berlin, Germany, 1993.

[2] G. G. Gundersen, E. M. Steinbart, and S. Wang, "The possible orders of solutions of linear differential equations with polynomial coefficients," Transactions of the American Mathematical Society, vol. 350, no. 3, pp. 1225-1247, 1998.

[3] S. Hellerstein, J. Miles, and J. Rossi, "On the growth of solutions of certain linear differential equations," Annales Academiae Scientiarum Fennicae. Series A, vol. 17, no. 2, pp. 343-365, 1992.

[4] J. Wang and I. Laine, "Growth of solutions of nonhomogeneous linear differential equations," Abstract and Applied Analysis, vol. 2009, Article ID 363927, 11 pages, 2009.

[5] G. G. Gundersen and E. M. Steinbart, "Finite order solutions of nonhomogeneous linear differential equations," Annales Academiae Scientiarum Fennicae. Series A, vol. 17, no. 2, pp. 327-341, 1992.

[6] J. Wang and I. Laine, "Growth of solutions of second order linear differential equations," Journal of Mathematical Analysis and Applications, vol. 342, no. 1, pp. 39-51, 2008.

[7] Z. X. Chen, "The fixed points and hyper order of solutions of second order complex differential equations," Acta Mathematica Scientia. Series A, vol. 20, no. 3, pp. 425-432, 2000, English translation in Chinese Journal of Contemporary Mathematics, vol. 27, no. 3, pp. 217-230, 2006.

[8] J. Wang and W. R. Lü, "The fixed points and hyper-order of solutions of second order linear differential equations with meromorphic coefficients," Acta Mathematicae Applicatae Sinica, vol. 27, no. 1, pp. 72-80, 2004.

[9] J. Wang and H.-X. Yi, "Fixed points and hyper order of differential polynomials generated by solutions of differential equation," Complex Variables, vol. 48, no. 1, pp. 83-94, 2003.

[10] M.-S. Liu and X.-M. Zhang, "Fixed points of meromorphic solutions of higher order linear differential equations," Annales Academix Scientiarium Fennicæ, vol. 31, no. 1, pp. 191-211, 2006.

[11] Z. X. Chen and K. H. Shon, "The relation between solutions of a class of second-order differential equations with functions of small growth," Chinese Annals of Mathematics Series A, vol. 27, no. 4, pp. 431-442, 2006.

[12] G. G. Gundersen, "Estimates for the logarithmic derivative of a meromorphic function, plus similar estimates," Journal of the London Mathematical Society, vol. 37, no. 1, pp. 88-104, 1988.

[13] A. I. Markushevich, Theory of Functions of a Complex Variable. Vol. II, Prentice-Hall, Englewood Cliffs, NJ, USA, 1965.

[14] Z. X. Chen, "Zeros of meromorphic solutions of higher order linear differential equations," Analysis, vol. 14, no. 4, pp. 425-438, 1994.

[15] C.-C. Yang and H.-X. Yi, Uniqueness Theory of Meromorphic Functions, vol. 557 of Mathematics and its Applications, Kluwer Academic Publishers, Dordrecht, The Netherlands, 2003.

[16] Z. X. Chen and K. H. Shon, "On the growth and fixed points of solutions of second order differential equations with meromorphic coefficients," Acta Mathematica Sinica, vol. 21, no. 4, pp. 753-764, 2005. 


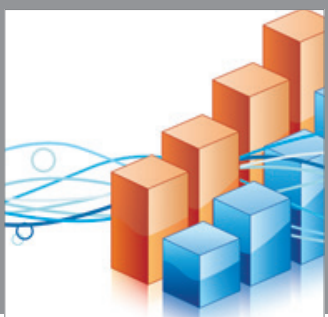

Advances in

Operations Research

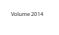

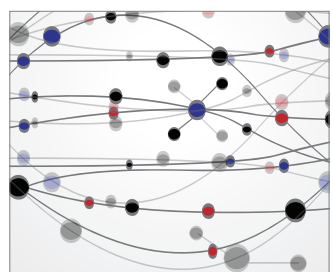

\section{The Scientific} World Journal
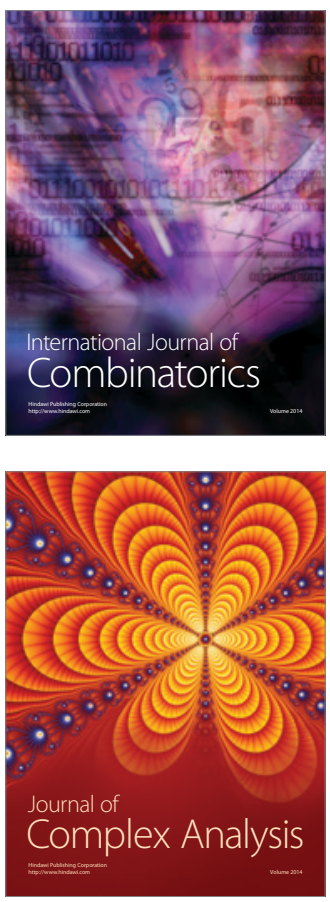

International Journal of

Mathematics and

Mathematical

Sciences
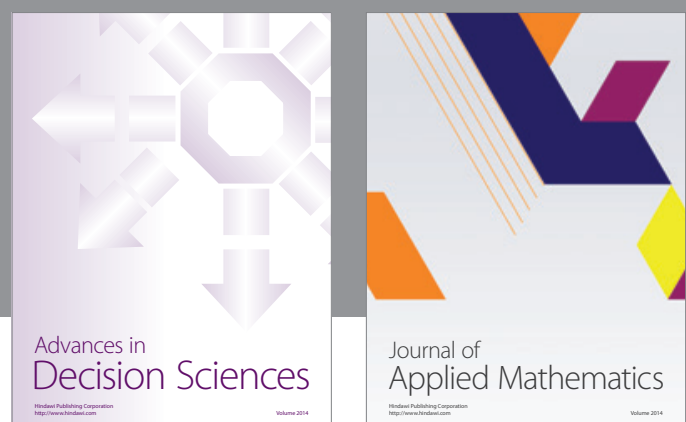

Journal of

Applied Mathematics
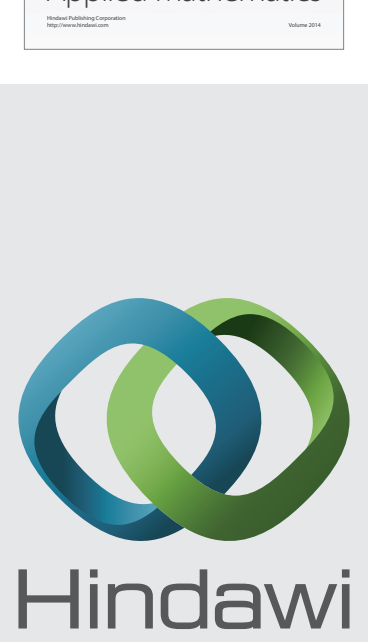

Submit your manuscripts at http://www.hindawi.com
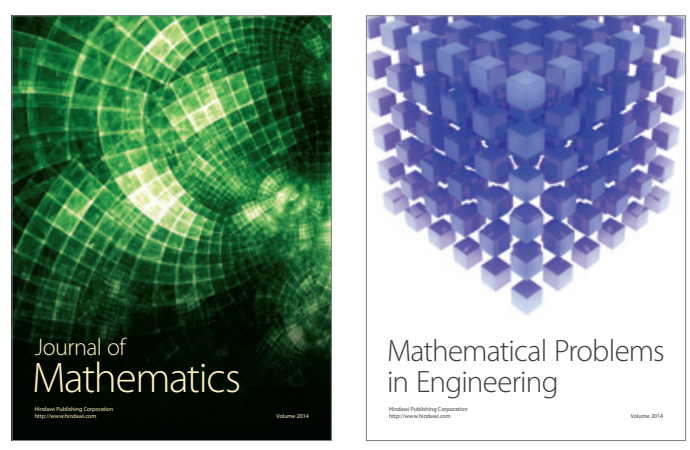

Mathematical Problems in Engineering
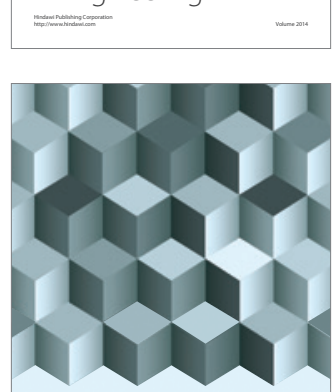

Journal of

Function Spaces
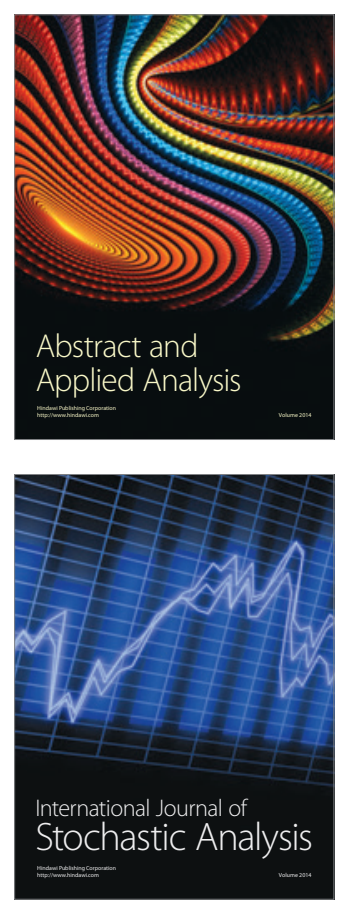

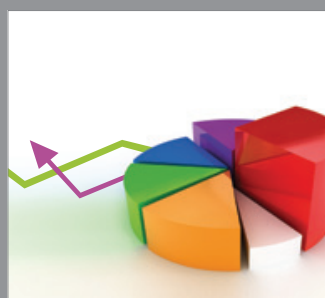

ournal of

Probability and Statistics

Promensencen
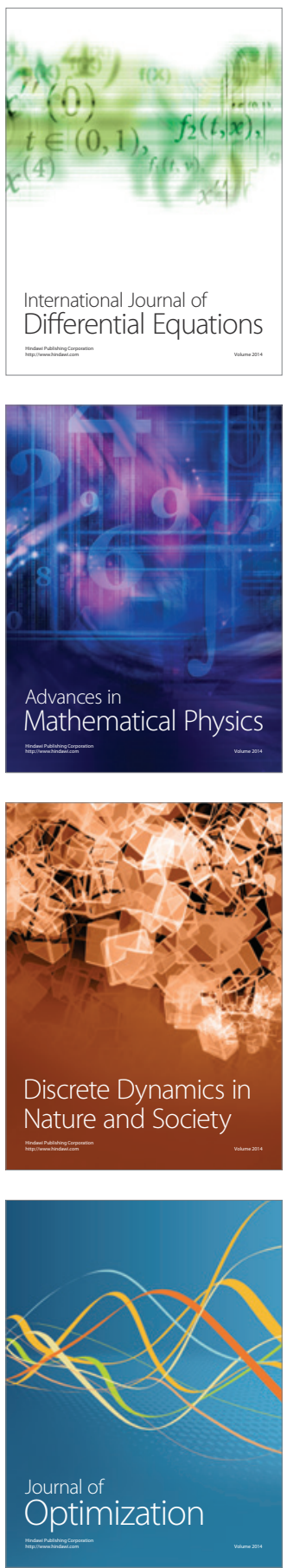